Pacific Journal of Mathematics

FXEE PONTS AND FRER M MPS 


\title{
FIXED POINTS AND FIBRE MAPS
}

\author{
ROBERT F. BROWN
}

Let $\mathscr{F}=(E, \boldsymbol{p}, \boldsymbol{B})$ be a (Hurewicz) fibre space and let $\lambda$ be a lifting function for $\mathscr{F}$. For $W$ a subset of $B$, a map $f: p^{-1}(W) \rightarrow E$ is called a fibre map if $p(e)=p\left(e^{\prime}\right)$ implies $p(f(e))=p\left(f\left(e^{\prime}\right)\right)$. Define $\bar{f}: W \rightarrow B$ to be the map such that $\bar{f} p=p f$. If $[W \cup \bar{f}(W)] \subseteq V \leqq B$ where $V$ is pathwise connected, define $f_{b}^{V}: p^{-1}(b) \rightarrow p^{-1}(b)$, for $b \in W$, by $f_{b}^{V}(e)=\lambda(f(e)$, $\omega)(1)$ where $\omega: I \rightarrow V$ is a path such that $\omega(0)=\bar{f}(b)$ and $\omega(1)=b$. Let $i$ be a fixed point index defined on the category of compact ANR's and let $Q$ denote the rationals. The main result of this paper is:

Theorem 1. Let $\mathscr{F}=(E, p, B)$ be a fibre space such that $E, B$, and all the fibres are compact ANR's. Let $f: E \rightarrow E$ be a fibre map. If $U$ is an open subset of $B$ such that $\bar{f}(b) \neq b$ for all $b \in b d(U)$ and $\operatorname{cl}[U \cup \bar{f}(U)] \subseteq V \cong \dot{B}$ where $V$ is open and pathwise connected and $\mathscr{F} \mid V=\left(p^{-1}(V), p, V\right)$ is $Q$-orientable, then

$$
i\left(f, p^{-1}(V)\right)=i(\bar{f}, U) . L\left(f_{b}^{V}\right)
$$

where $L\left(f_{b}^{V}\right)$ is the Lefschetz number of $f_{b}^{V}$ for any $b \in U$.

Independence of $L\left(f_{b}^{V}\right)$. For $\mathscr{F}=(E, p, B)$ a Hurewicz fibre space with lifting function $\lambda$ [7] and $\omega$ a loop in $B$ based at $b$, define $\varphi: p^{-1}(b) \rightarrow p^{-1}(b)$ by $\varphi(e)=\lambda(e, \omega)(1)$. The fibre space $\mathscr{F}$ is called Q-orientable if

$$
\varphi^{*}: H^{*}\left(p^{-1}(b) ; Q\right) \rightarrow H^{*}\left(p^{-1}(b) ; Q\right)
$$

is the identity isomorphism for all pairs $(b, \omega)$ where $b \in B$ and $\omega$ is a loop in $B$ based at $b$.

Lemma. Let $\mathscr{F}=(E, p, B)$ be a Q-orientable fibre space and let $\omega_{i}: I \rightarrow B, i=1,2$, be paths such that $\omega_{i}(0)=b$ and $\omega_{i}(1)=b^{\prime}$. Define $\varphi_{i}: p^{-1}(b) \rightarrow p^{-1}\left(b^{\prime}\right)$ by $\varphi_{i}(e)=\lambda\left(e, \omega_{i}\right)(1)$, then

$$
\varphi_{1}^{*}=\varphi_{2}^{*}: H^{*}\left(p^{-1}\left(b^{\prime}\right) ; Q\right) \stackrel{\cong}{\cong} H^{*}\left(p^{-1}(b) ; Q\right) \text {. }
$$

Proof. By Proposition 2 of [4], each $\varphi_{i}$ is a homotopy equivalence with homotopy inverse $\psi_{i}: p^{-1}\left(b^{\prime}\right) \rightarrow p^{-1}(b)$ given by $\psi_{i}\left(e^{\prime}\right)=\lambda\left(e^{\prime}, \bar{\omega}_{i}\right)(1)$ where $\bar{\omega}_{i}(s)=\omega_{i}(1-s)$. Therefore, $\varphi_{i}^{*}: H^{*}\left(p^{-1}\left(b^{\prime}\right) ; Q\right) \rightarrow H^{*}\left(p^{-1}(b) ; Q\right)$ is an isomorphism and $\psi_{i}^{*}=\left(\varphi_{i}^{*}\right)^{-1}$. Consider $\omega: I \rightarrow B$ defined by

$$
\omega(s)= \begin{cases}\omega_{1}(2 s) & 0 \leqq s \leqq 1 / 2 \\ \bar{\omega}_{2}(1-2 s) & 1 / 2 \leqq s \leqq 1\end{cases}
$$


then $\omega$ is a loop in $B$ based at $b$ and since $\mathscr{F}$ is $Q$-orientable, for $\varphi(e)=\lambda(e, \omega)(1), \varphi^{*}$ is the identity isomorphism. It follows from [4] that $\varphi$ is homotopic to $\psi_{2} \varphi_{1}$ so $\varphi^{*}=\varphi_{1}^{*} \psi_{2}^{*}$ and $\psi_{2}^{*}=\left(\varphi_{1}^{*}\right)^{-1}$. Hence $\psi_{2}^{*}=\psi_{1}^{*}$ and $\varphi_{2}^{*}=\varphi_{1}^{*}$.

THeorem 2. Let $\mathscr{F}=(E, p, B)$ be a Q-orientable fibre space where $B$ is pathwise connected and $H^{*}\left(p^{-1}(b) ; Q\right)$ is finitely generated for $b \in B$. For $W \cong B$, let $f: p^{-1}(W) \rightarrow E$ be a fibre map, then $L\left(f_{b}\right)=L\left(f_{b^{\prime}}\right)$ for all $b, b^{\prime} \in W$, where $f_{b}$ means $f_{b}^{B}$.

Proof. Since $f_{b}=\varphi_{i}\left(f \mid p^{-1}(b)\right)$, the lemma implies that

$$
f_{b}^{*}: H^{*}\left(p^{-1}(b) ; Q\right) \rightarrow H^{*}\left(p^{-1}(b) ; Q\right)
$$

is independent of the choice of the path $\omega_{i}$ from $\bar{f}(b)$ to $b$. Let $\omega_{0}, \omega_{1}: I \rightarrow B$ such that $\omega_{0}(0)=\bar{f}(b), \omega_{0}(1)=\omega_{1}(0)=b$, and $\omega_{1}(1)=b^{\prime}$. Define $\omega_{2}: I \rightarrow B$ by

$$
\omega_{2}(s)= \begin{cases}\overline{f\left(\omega_{1}\right.}(2 s) & 0 \leqq s \leqq 1 / 2 \\ \omega_{0}(2 s-1) & 1 / 2 \leqq s \leqq 1\end{cases}
$$

We first show that diagram (1) is homotopy commutative, where $\varphi_{i}(e)=\lambda\left(e, \omega_{\imath}\right)(1), i=0,1,2$.

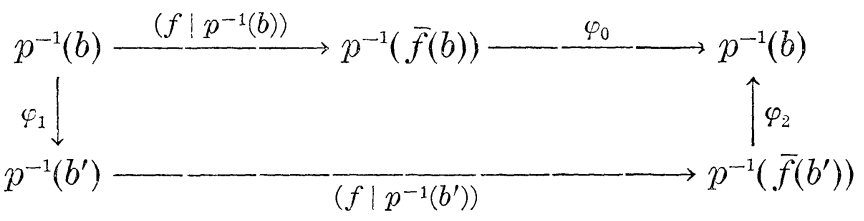

Define the homotopy $H: p^{-1}(b) \times I \rightarrow p^{-1}(b)$ by

$$
H(e, t)=\lambda\left[f\left(\lambda\left(e, \omega_{1}\right)(1-t)\right), \omega^{t}\right](1)
$$

where

$$
\omega^{t}(s)= \begin{cases}\bar{f}\left(\bar{\omega}_{1}(2 s+t)\right) & 0 \leqq s \leqq \frac{1-t}{2} \\ \omega_{0}\left(\frac{2 s+t-1}{t+1}\right) & \frac{1-t}{2} \leqq s \leqq 1\end{cases}
$$

Then $H(e, 0)=\varphi_{2} f \varphi_{1}(e)$ and $H(e, 1)=\varphi_{0} f(e)$ as required. By the lemma and [4], $\left(f_{b^{\prime}}\right)^{*}=\left(\varphi_{1} \varphi_{2}\left(f \mid p^{-1}\left(b^{\prime}\right)\right)\right)^{*}$. Furthermore,

$$
\begin{aligned}
\left(\psi_{1} f_{b^{\prime}}, \varphi_{1}\right)^{*} & =\left(\psi_{1}^{\prime} \varphi_{1} \varphi_{2}\left(f \mid p^{-1}\left(b^{\prime}\right)\right) \varphi_{1}\right)^{*} \\
& =\left(\varphi_{2}\left(f \mid p^{-1}\left(b^{\prime}\right)\right) \varphi_{1}\right)^{*}=\left(\varphi_{0}\left(f \mid p^{-1}(b)\right)\right)^{*}=f_{b}^{*} .
\end{aligned}
$$

Since $Q$ is a field, $H^{*}\left(p^{-1}(b) ; Q\right)$ and $H^{*}\left(p^{-1}\left(b^{\prime}\right) ; Q\right)$ are finite dimensional 
vector spaces and $\varphi_{1}^{*}, f_{b^{\prime}}^{*}, \psi_{1}^{*}$ are linear transformations. Pick bases for $H^{k}\left(p^{-1}(b) ; Q\right)$ and $H^{k}\left(p^{-1}\left(b^{\prime}\right) ; Q\right)$ and let $\Phi, F^{\prime}$, and $\Psi$ be the matrices with respect to these bases representing $\varphi_{1}^{*, k}, f_{b}^{*, k}$, and $\psi_{1}^{*, t c}$ respectively. Since $\psi_{1}^{*}=\left(\varphi_{1}^{*}\right)^{-1}, \Psi \Phi=E_{n}$, the $n \times n$ identity matrix, where $n$ is the dimension of $H^{k}\left(p^{-1}(b) ; Q\right)$. Therefore, trace $\left(\Phi F^{\prime} \Psi\right)=$ trace $\left(F^{\prime}\right)$ which implies that $L\left(f_{b^{\prime}}\right)=L\left(\psi_{1} f_{b}, \varphi_{1}\right)$. The theorem now follows because $\left(\psi_{1} f_{b}, \varphi_{1}\right)^{*}=f_{b}^{*}$ implies $L\left(\psi_{1} f_{b}, \varphi_{1}\right)=L\left(f_{b}\right)$.

2. Extension of a theorem of Leray. Let $B$ and $F$ be topological spaces and let $\left(B \times F, \pi^{1}, B\right)$ be the trivial fibre space. Suppose $W$ is a subset of $B$ and $f: W \times F \rightarrow B \times F$ is a fibre map. Define $f_{b}: F \rightarrow F$ by $f_{b}=\pi^{2} f j_{b}$ where $j_{b}: F \rightarrow W \times F$ is given by $j_{b}(x)=(b, x)$ and $\pi^{2}: B \times F \rightarrow F$ is projection. Theorem 3 is a restatement of Theorem 27 of [9] in the somewhat specialized form in which we shall use it.

THEOREM 3 (Leray). Let $\left(B \times F, \pi^{1}, B\right)$ be the trivial fibre space where $B$ and $F$ are finite polyhedra. For $U$ an open connected subset of $B$, let $f: \operatorname{cl}(U) \times F \rightarrow B \times F$ be a fibre map. ${ }^{1}$ If $\bar{f}(b) \neq b$ for all $b \in b d(U)$, then

$$
\bar{i}(f, U \times F)=\bar{i}(\bar{f}, U) \cdot L\left(f_{b}\right)
$$

for all $b \in U$, where $\bar{i}$ denotes the Leray fixed point index.

By Theorem 22 and Corollary 26-27 of [9], the Leray index [9, p. 208] satisfies the O'Neill axioms [10, p. 500]. (We will use the formulation of the axioms and the terminology of [1]). Therefore, an index $i$ for the category of compact ANR's, satisfying the O'Neill axioms, may be obtained from the index $\bar{i}$ in the following manner [2, p. 20]. Let $X$ be a compact ANR and let $\alpha$ be a finite open cover of $X$, then there exists a finite polyhedron $K$ and maps $\varphi: X \rightarrow K$, $\psi: K \rightarrow X$ such that $\psi \varphi$ is $\alpha$-homotopic to the identity map on $X$, i.e. there exists a map $H: X \times I \rightarrow X$ such that $H(x, 0)=x, H(x, 1)=$ $\psi \varphi(x)$, and for each $x \in X$, the set $\{H(x, t) \mid t \in I\}$ lies in a single element of $\alpha$ [5, Theorem 6.1]. Write $\psi \varphi \sim_{\alpha} 1_{x}$. For $U$ an open subset of $X$ and $f: X \rightarrow X$ a map such that $f(x) \neq x$ for all $x \in b d(U)$, let

$$
i_{\alpha}(f, U)=\bar{i}\left(\varphi f \psi, \psi^{-1}(U)\right) \text {. }
$$

Browder [2, Theorem 2, p. 20] showed that there exists a finite open cover $\kappa_{f}(U)$ of $X$ such that if $\alpha$ is a refinement of $\kappa_{f}(U)$, then $i_{\alpha}(f, U)$ is well-defined and independent of $\alpha, \varphi$, and $\psi$. Write $i_{\alpha}=i$ for all

1 The notation $\operatorname{cl}(U)$ denotes the closure of $U$. We use bd( $U)$ for the boundary of $U$. 
such $\alpha$.

THEOREM 4. Let $\left(B \times F, \pi^{1}, B\right)$ be the trivial fibre space where $B$ is a finite polyhedron and $F$ is a compact ANR. For $U$ a connected open subset of $B$, let $f: \operatorname{cl}(U) \times F \rightarrow B \times F$ be a fibre map. If $\bar{f}(b) \neq b$ for all $b \in b d(U)$, then

$$
i(f, U \times F)=\bar{i}(\bar{f}, U) \cdot L\left(f_{b}\right)
$$

for all $b \in U$.

Proof. Let $F$ be dominated by a finite polyhedron $K$ by means of maps $\varphi: F \rightarrow K$ and $\psi: K \rightarrow F$. Define $f^{*}: B \times K \rightarrow B \times K$ by $f^{\sharp}(b, k)=\left(\bar{f}(b), \varphi f_{b} \psi(k)\right)$ then $f^{\#}$ is a fibre map with respect to $\left(B \times K, \pi^{1}, B\right)$ and $\bar{f}^{*}=\bar{f}$. Since $\psi \varphi$ is homotopic to the identity map on $F, L\left(f_{b}^{\sharp}\right)=L\left(f_{b}\right)$ (see the proof that $L\left(f_{b^{\prime}}\right)=L\left(\psi_{1} f_{b^{\prime}}, \varphi_{1}\right)$ in Theorem 2). Let $\alpha$ be a finite open cover of $B$ which refines $\kappa_{\bar{f}}(U)$, then $\tau=\left\{\left(\pi^{1}\right)^{-1}(A) \mid A \in \alpha\right\}$ refines $\kappa_{f}\left(p^{-1}(U)\right)$. Since $f^{\sharp}=\left(1_{B} \times \varphi\right) f\left(1_{B} \times \psi\right)$ and, trivially,

$$
\left(1_{B} \times \psi\right)\left(1_{B} \times \varphi\right) \sim_{\tau} 1_{B} \times 1_{F},
$$

then $i(f, U \times F)=\bar{i}\left(f^{\sharp}, U \times K\right)$. Therefore, by Theorem 3,

$$
i(f, U \times F)=\bar{i}(\bar{f}, U) \cdot L\left(f_{b}\right) .
$$

3. Proof of Theorem 1. We first assume that $B$ is a finite polyhedron. By a theorem of Hopf [6, Theorem 5], given $\varepsilon>0$, there exists a map $\bar{g}: B \rightarrow B$ homotopic to $\bar{f}$ by a homotopy $h: B \times I \rightarrow B$ such that $h(b, 0)=\bar{f}(b), h(b, 1)=\bar{g}(b)$ and $\rho\left[h(b, t), h\left(b, t^{\prime}\right)\right]<\varepsilon$ for $b \in B$, $t, t^{\prime} \in I$, where $\rho$ is the metric of $B$. The map $\bar{g}$ has a finite number of fixed points $b_{1}, \cdots, b_{s}$ where, with respect to some barycentric subdivision of $B$, each $b_{j}$ lies in the interior of a different simplex $\sigma_{j}$ of $B$, where $\sigma_{j}$ is not a face of any other simplex of $B$. Since $\bar{f}$ has no fixed points on $b d(U)$, inf $\{\rho(b, f(b)) \mid b \in b d(U)\}=\varepsilon_{1}>0$. Let $\varepsilon_{2}>0$ be the distance from cl $[U \cup \bar{f}(U)]$ to $B-V$ (if $V=B$, take $\varepsilon_{2}=\infty$ ). Let $\varepsilon=\min \left(\varepsilon_{1}, \varepsilon_{2}\right)$ then $h(b, t) \neq b$ for all $b \in b d(U)$. Hence $i(\bar{f}, U)=$ $i(\bar{g}, U)$ by the homotopy axiom. Furthermore, cl $[U \cup \bar{g}(U)] \leqq V$. The homotopy $h$ induces $h^{\prime}: B \rightarrow B^{I}$. Let $\lambda$ be regular lifting function for $\mathscr{F}$ and define $H^{\prime}: E \rightarrow E^{I}$ by

$$
H^{\prime}(e)(t)=\lambda\left(f(e), h^{\prime}(p(e))\right)(t) .
$$

Define $g: E \rightarrow E$ by $g(e)=H^{\prime}(e)(1)$ then $g$ is a fibre map homotopic to $f$ by a homotopy without fixed points on $b d\left(p^{-1}(U)\right)$ so $i\left(f, p^{-1}(U)\right)=$ $i\left(g, p^{-1}(U)\right)$. Furthermore, $p g=\bar{g} p$. Since $f_{b_{j}}$ is precisely $g_{b_{j}}$ if we use the path $h^{\prime}\left(b_{j}\right)$ to define $f_{b_{j}}$ and the constant path to define $g_{b_{j}}$, 
then $L\left(f_{b_{j}}^{V}\right)=L\left(g_{b_{j}}^{V}\right)$. We have shown that when $B$ is a finite polyhedron, it is sufficient to verify the conclusion for the map $g$.

Let $U_{j}$ be a $\delta$-neighborhood of $b_{j}$ where $\delta$ is chosen small enough so that $\left[\mathrm{cl}\left(U_{j}\right) \cup \bar{g}\left(\mathrm{cl}\left(U_{j}\right)\right)\right] \subseteq \sigma_{j}$. We may contract $\sigma_{j}$ to $b_{j}$ so that $b_{j}$ stays fixed throughout the contraction and such that the restriction to $\mathrm{cl}\left(U_{j}\right)$ contracts $\mathrm{cl}\left(U_{j}\right)$ through itself to $b_{j}$. The contraction induces fibre homotopy equivalences

$$
\begin{aligned}
\alpha: p^{-1}\left(\sigma_{j}\right) & \rightleftarrows \sigma_{j} \times F: \beta \\
\alpha^{\prime}: p^{-1}\left(\operatorname{cl}\left(U_{j}\right)\right) & \rightleftarrows \operatorname{cl}\left(U_{j}\right) \times F: \beta^{\prime}
\end{aligned}
$$

where the primes denote restriction and $F=p^{-1}\left(b_{j}\right)$ [4, Proposition 4]. Consider the diagram

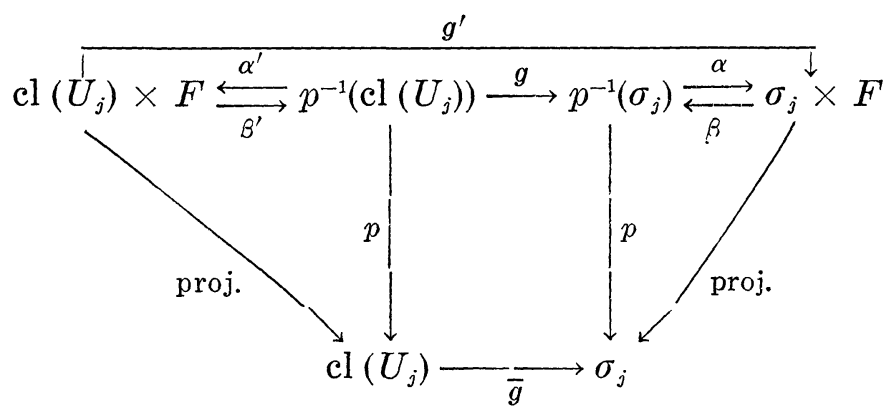

where $g^{\prime}=\alpha g \beta^{\prime} . \quad$ By Theorem 4,

$$
i\left(g^{\prime}, U_{j} \times F\right)=\bar{i}(\bar{g}, U) \cdot L\left(g_{b_{j}}^{\prime}\right) .
$$

If we use the constant path to define $g_{b_{j}}$, then $g_{b_{j}}=g_{b_{j}}^{\prime}$, so $L\left(g_{b}^{F}\right)=$ $L\left(g_{b_{j}}^{\prime}\right)$. Let $\mu=g \beta^{\prime}: p^{-1}\left(\operatorname{cl}\left(U_{j}\right)\right) \rightarrow \sigma_{j} \times F$, then by the commutativity axiom

$$
i\left(\alpha \mu, U_{j} \times F\right)=i\left(\mu \alpha^{\prime}, p^{-1}\left(U_{j}\right)\right) .
$$

Now $i\left(\alpha \mu, U_{j} \times F\right)=i\left(g^{\prime}, U_{j} \times F\right)$ by definition. On the other hand, $\mu \alpha^{\prime}=g \beta^{\prime} \alpha^{\prime}$ is homotopic to $g$ by a homotopy which has no fixed points on $b d\left(p^{-1}\left(U_{j}\right)\right)$ since $\bar{g}$ has no fixed points on $b d\left(U_{j}\right)$ and the homotopy between $\beta^{\prime} \alpha^{\prime}$ and the identity is fibre-preserving, so by the homotopy axiom $i\left(\mu \alpha^{\prime}, p^{-1}\left(U_{j}\right)\right)=i\left(g, p^{-1}\left(U_{j}\right)\right)$. Therefore

$$
i\left(g, p^{-1}\left(U_{j}\right)\right)=\bar{i}\left(\bar{g}, U_{j}\right) \cdot L\left(g_{b}^{V}\right) .
$$

Renumber the fixed points of $\bar{g}$ so that $b_{1}, \cdots, b_{q}$ are the fixed points which lie in $U$. Since $g(e)=e$ implies $p(e)=b_{j}$ for some $j=$ $1, \cdots, s, g$ has no fixed points on $\left[p^{-1}(\operatorname{cl}(U))-\bigcup_{j=1}^{q} p^{-1}\left(U_{j}\right)\right]$. Hence by the additivity axiom, 


$$
\begin{aligned}
i\left(g, p^{-1}(U)\right) & =\sum_{j=1}^{q} i\left(g, p^{-1}\left(U_{j}\right)\right) \\
& =\sum_{j=1}^{q} \bar{i}\left(\bar{g}, U_{j}\right) L\left(g_{b}^{V}\right)=\bar{i}(\bar{g}, U) \cdot L\left(g_{b}^{V}\right) .
\end{aligned}
$$

Now suppose that $B$ is a compact ANR, let $K$ be a finite polyhedron and let $\varphi: B \rightarrow K, \psi: K \rightarrow B$ be maps such that $\psi \varphi \sim_{\alpha} 1_{\beta}$ where $\alpha$ refines $\kappa_{\bar{f}}(U)$ and $\alpha(\bar{f}(U))$, the union of all $A \in \alpha$ such that $A \cap \bar{f}(U) \neq$ $\varnothing$, is contained in $V$. Let $\psi^{*}(\mathscr{F})=\left(\psi^{*}(E), p^{*}, K\right)$ where

$$
\psi^{\sharp}(E)=\{(k, e) \in K \times E \mid \psi(k)=p(e)\}
$$

and $p^{*}(k, e)=k$, then $\psi^{*}(F)$ is a fibre space with lifting function $\lambda^{*}$ given by

$$
\lambda^{\sharp}((k, e), \omega)(t)=(\omega(t), \lambda(e, \psi \omega)(t))
$$

where $\lambda$ is the lifting function of $\mathscr{F}$. Let $h: B \times I \rightarrow B$ be the $\alpha$-homotopy such that $h(b, 0)=b, h(b, 1)=\psi(b)$, then $h$ induces $h^{\prime}: B \rightarrow B^{I}$. Define $\varphi^{\prime}: E \rightarrow \gamma^{\sharp}(E)$ by

$$
\varphi^{\prime}(e)=\left(\varphi p(e), \lambda\left(e, h^{\prime}(p(e))\right)(1)\right.
$$

Consider

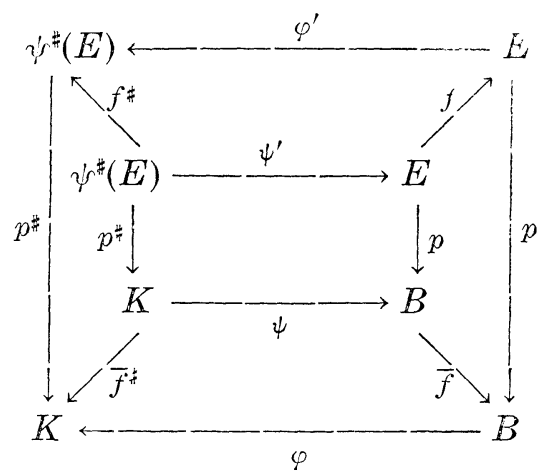

where $\psi^{\prime}(k, e)=e$ and $f^{\sharp}=\varphi^{\prime} f \psi^{\prime}$. Since $\overline{f^{*}}=\varphi \bar{f} \psi$ and $\psi \varphi \sim_{\alpha} 1_{B}$, then $i(\bar{f}, U)=\bar{i}\left(\bar{f}^{\sharp}, \psi^{-1}(U)\right)$. We let $\nu=\phi^{\prime} f: E \rightarrow \psi^{*}(E)$, then by the commutativity axiom,

$$
i\left(\psi^{\prime} \nu, p^{-1}(U)\right)=i\left(\nu \psi^{\prime}, \psi^{\prime-1} p^{-1}(U)\right) .
$$

Define $H: E \times I \rightarrow E$ by $H(e, t)=\lambda\left(e, h^{\prime}(p(e))\right)(t)$. If $H(f(e), t)=e$ for any $e \in b d\left(p^{-1}(U)\right), t \in I$, then $h(\bar{f}(p(e)), t)=p(e)$ which is impossible since $\alpha$ refines $\kappa_{\bar{f}}(U)\left\lfloor 2\right.$, p. 20], so $\psi^{\prime} \nu=\psi^{\prime} \varphi^{\prime} f$ is homotopic to $f$ by a homotopy without fixed points on $b d\left(p^{-1}(U)\right)$ and by the homotopy axiom

$$
i\left(\psi^{\prime} \nu, p^{-1}(U)\right)=i\left(f, p^{-1}(U)\right)
$$


On the other hand, $i\left(\nu \psi^{\prime}, \psi^{\prime-1} p^{-1}(U)\right)=i\left(f^{\sharp}, p^{\sharp-1}\left(\psi^{-1}(U)\right)\right)$. If $k \in \psi^{-1}(U)$, then $\bar{f}^{\sharp}(k) \in \psi^{-1}(V)=W$ since $\alpha(\bar{f}(U)) \subseteq V$. Let $\omega: I \rightarrow W$ be a path such that $\omega(0)=\bar{f}^{\sharp}(k)$ and $\omega(1)=k$. Define $\omega^{\prime}: I \rightarrow V$ by

$$
\omega^{\prime}(s)= \begin{cases}h^{\prime}(\bar{f} \psi(k))(2 s) & 0 \leqq s \leqq 1 / 2 \\ \psi \omega(2 s-1) & 1 / 2 \leqq s \leqq 1\end{cases}
$$

and let $f_{\psi(k)}$ be given by $f_{\psi(k)}(e)=\lambda\left(f(e), \omega^{\prime}\right)(1)$. Define $f_{\psi(k)}^{\prime}: p^{-1}(\psi(k)) \rightarrow$ $p^{-1}(\psi(k))$ by

$$
f_{\psi(k)}^{\prime}(e)=\lambda\left[\lambda\left(f(e), h^{\prime}(\bar{f} \psi(k))\right)(1), \psi \omega\right](1),
$$

then by [4], $f_{\psi(k)}^{\prime}$ is homotopic to $f_{\psi(k)}$. But $f_{k}^{\sharp}(k, e)=\lambda^{\sharp}((k, e), \omega)(1)=$ $\left(k, f_{\psi(k)}^{\prime}(e)\right)$. Therefore $L\left(f_{k}^{\sharp W}\right)$ is equal to $L\left(f_{b}^{V}\right)$ and is independent of $k$ and $\omega$. Applying the first part of the proof to the fibre space $\psi^{*}(\mathscr{F})$, the map $f^{*}$, and the open set $\psi^{-1}(U) \subseteq K$, we get

$$
i\left(f^{\sharp}, p^{\sharp-1}\left(\psi^{-1}(U)\right)\right)=\bar{i}\left(\bar{f}^{\sharp}, \psi^{-1}(U)\right) \cdot L\left(f_{k}^{\sharp W}\right) \text {. }
$$

Therefore,

$$
i\left(f, p^{-1}(U)\right)=i(\bar{f}, U) \cdot L\left(f_{b}^{V}\right)
$$

which completes the proof of Theorem 1 .

4. The index of a fixed point class. Let $X$ be a compact ANR and let $f: X \rightarrow X$ be a map. Denote the fixed point classes of $f$ by $\boldsymbol{F}_{1}, \cdots, \boldsymbol{F}_{r}$. Let $(\tilde{X}, \widetilde{p}, X)$ be the universal covering space of $X$, then by [2, pp. 43-44] there is a map $\tilde{f}^{j}: \widetilde{X} \rightarrow \widetilde{X}$ such that $\widetilde{p} \tilde{f}^{j}=f \widetilde{p}$ which has the following properties: (1) if $\tilde{f}^{j}(e)=e$, then $p(e) \in \boldsymbol{F}_{j}$, (2) for each $b \in \boldsymbol{F}_{j}$ there exists $e \in \widetilde{p}^{-1}(b)$ such that $\tilde{f}^{j}(e)=e$. We say that $\tilde{f}^{j}$ covers $\boldsymbol{F}_{j}$. There is an open set $U_{j}$ in $X$ containing $\boldsymbol{F}_{j}$ such that $\operatorname{cl}\left(U_{j}\right) \cap \boldsymbol{F}_{k}=\varnothing$ for $k \neq j$. The index of $\boldsymbol{F}_{j}$ is defined by $i\left(\boldsymbol{F}_{j}\right)=$ $i\left(f, U_{j}\right)$ and is independent of the choice of $U_{j}$.

THEOREM 5. Let $X$ be a compact ANR with finite fundamental group. Let $f: X \rightarrow X$ be a map, let $\boldsymbol{F}$ be a fixed point class of $f$, and let $\tilde{f}: \widetilde{X} \rightarrow \widetilde{X}$ cover $\boldsymbol{F}$. If there exists an open subset $U$ of $X$ such that for $x \in U, f(x)=x$ if, and only if, $x \in \boldsymbol{F}, f(x) \neq x$ for $x \in b d(U)$, and $\operatorname{cl}[U \cup f(U)] \subseteq V$, where $V$ is an open connected simply-connected subset of $X$, then

$$
i(\boldsymbol{F})=L(\widetilde{f}) / L\left(\tilde{f}_{x}^{V}\right)
$$

for $x \in U$.

Proof. We first observe that $L\left(\widetilde{f_{x}^{V}}\right) \neq 0$. Take $x \in \boldsymbol{F}$, then since the fibre is discrete $L\left(\tilde{f}_{x}^{V}\right)$ is just the number of fixed points of $\tilde{f}$ 
restricted to $\widetilde{p}^{-1}(x)$ which, since $\tilde{f}$ covers $\boldsymbol{F}$, must be greater than zero. Since $\pi_{1}(X)$ is finite, $\tilde{X}$ is compact and we can apply Theorem 1 to obtain

$$
i(f, U)=i\left(\tilde{f}, \widetilde{p}^{-1}(U)\right) / L\left(\tilde{f}_{x}^{V}\right) .
$$

Since $\tilde{f}$ has no fixed points outside of $\widetilde{p}^{-1}(U), i\left(\tilde{f}, \widetilde{p}^{-1}(U)\right)=L(\tilde{f})$.

The existence of the simply-connected set $V$ in the hypotheses of Theorem 5 is not as severe a restriction as it may appear. For example, if $X$ is a finite polyhedron, (or a compact topological manifold, with or without boundary) $f$ is homotopic to a map $g$ which has only isolated fixed points [6, Theorem 5] [3, Theorem 2] and the homotopy carries $\boldsymbol{F}$ to a fixed point class $\boldsymbol{F}^{\prime}$ of $g$ of the same index [2, Theorem 3 , p. 36]. Hence we can apply Theorem 5 to $g$ and $\boldsymbol{F}^{\prime}$ to compute $i(\boldsymbol{F})$ (compare Theorem 5.2 of [8]).

\section{REFERENCES}

1. F. Browder, On the fixed point index for continuous mappings of locally connected spaces, Summa Brasil. Math. 4 (1960), 253-293.

2. - The topological fixed point index and its application in functional analysis, Doctoral Dissertation, Princeton U., 1948.

3. R. Brown, On a homotopy converse to the Lefschetz fixed point theorem, Pacific J. Math. 17 (1966), 407-411.

4. E. Fadell, On fibre spaces, Trans. Amer. Math. Soc. 90 (1959), 1-14.

5. O. Hanner, Some theorems on absolute neighborhood retracts, Ark. Mat. 1 (1951), 389-408.

6. H. Hopf. Über die algebraische Anzahl von Fixpunkten, Math. Z. 29 (1929), 493-524.

7. W. Hurewicz, On the concept of a fibre space, Proc. Nat. Acad. Sci. U.S.A. 41 (1955), 956-961.

8. Bo-ju Jiang, Estimation of the Nielson numbers, Chinese Math 5 (1964), 330-339.

9. J. Leray, Sur les équations et les transformations, J. Math. Pures appl. (9 me série) 24 (1945), 201-248.

10. B. O'Neill, Essential sets and fixed points, Amer. J. Math. 75 (1953), 497-509.

Received June 15, 1965. This research was supported in part by the Air Force Office of Scientific Research and by the National Science Foundation.

UNiversity of California, Los ANGEles 


\section{PACIFIC JOURNAL OF MATHEMATICS}

\section{EDITORS}

\section{H. SAMELSON}

Stanford University

Stanford, California

\author{
J. P. JANS \\ University of Washington \\ Seattle, Washington 98105
}

\section{J. DuGUNDJI}

University of Southern California

Los Angeles, California 90007

RichaRd ARENS

University of California

Los Angeles, California 90024

\section{ASSOCIATE EDITORS}

E. F. BECKENBACH
B. H. NeUManN

F. WOLF

K. YOSIDA

\section{SUPPORTING INSTITUTIONS}

UNIVERSITY OF BRITISH COLUMBIA

CALIFORNIA INSTITUTE OF TECHNOLOGY

UNIVERSITY OF CALIFORNIA

MONTANA STATE UNIVERSITY

UNIVERSITY OF NEVADA

NEW MEXICO STATE UNIVERSITY

OREGON STATE UNIVERSITY

UNIVERSITY OF OREGON

OSAKA UNIVERSITY

UNIVERSITY OF SOUTHERN CALIFORNIA
STANFORD UNIVERSITY

UNIVERSITY OF TOKYO

UNIVERSITY OF UTAH

WASHINGTON STATE UNIVERSITY

UNIVERSITY OF WASHINGTON

AMERICAN MATHEMATICAL SOCIETY

CHEVRON RESEARCH CORPORATION

TRW SYSTEMS

NAVAL ORDNANCE TEST STATION

Mathematical papers intended for publication in the Pacific Journal of Mathematics should be typewritten (double spaced). The first paragraph or two must be capable of being used separately as a synopsis of the entire paper. It should not contain references to the bibliography. Manuscripts may be sent to any one of the four editors. All other communications to the editors should be addressed to the managing editor, Richard Arens at the University of California, Los Angeles, California 90024.

50 reprints per author of each article are furnished free of charge; additional copies may be obtained at cost in multiples of 50 .

The Pacific Journal of Mathematics is published monthly. Effective with Volume 16 the price per volume ( 3 numbers) is $\$ 8.00$; single issues, $\$ 3.00$. Special price for current issues to individual faculty members of supporting institutions and to individual members of the American Mathematical Society: $\$ 4.00$ per volume; single issues $\$ 1.50$. Back numbers are available.

Subscriptions, orders for back numbers, and changes of address should be sent to Pacific Journal of Mathematics, 103 Highland Boulevard, Berkeley 8, California.

Printed at Kokusai Bunken Insatsusha (International Academic Printing Co., Ltd.), No. 6, 2-chome, Fujimi-cho, Chiyoda-ku, Tokyo, Japan.

PUBLISHED BY PACIFIC JOURNAL OF MATHEMATICS, A NON-PROFIT CORPORATION

The Supporting Institutions listed above contribute to the cost of publication of this Journal, but they are not owners or publishers and have no responsibility for its content or policies. 


\section{Pacific Journal of Mathematics}

\section{Vol. 21, No. $3 \quad$ BadMonth, 1967}

Richard Allen Askey, A transplantation theorem for Jacobi coefficients . . . 393

Raymond Balbes, Projective and injective distributive lattices .......... 405

Raymond Balbes and Alfred Horn, Order sums of distributive lattices . . . . 421

Donald Charles Benson, Nonconstant locally recurrent functions ........ 437

Allen Richard Bernstein, Invariant subspaces of polynomially compact operators on Banach space ............................... 445

Robert F. Brown, Fixed points and fibre ................... 465

David Geoffrey Cantor, On the Stone-Weierstrass approximation theorem for valued fields ................................ 473

James Walton England, Stability in topological dynamics .............. 479

Alessandro Figà-Talamanca and Daniel Rider, A theorem on random

Fourier series on noncommutative groups.................. 487

Sav Roman Harasymiv, A note of dilations in $L^{p} \ldots \ldots \ldots \ldots \ldots \ldots . \ldots 493$

J. G. Kalbfleisch, A uniqueness theorem for edge-chromatic graphs ....... 503

Richard Paul Kelisky and Theodore Joseph Rivlin, Iterates of Bernstein polynomials .................................... 511

D. G. Larman, On the union of two starshaped sets ............... 521

Henry B. Mann, Josephine Mitchell and Lowell Schoenfeld, Properties of differential forms in $n$ real variables ...................... 525

John W. Moon and Leo Moser, Generating oriented graphs by means of team comparisons .

Veikko Nevanlinna, A refinement of Selberg's asymptotic equation ...

Ulrich Oberst, Relative satellites and derived functors of functors with additive domain ..............................

John Vincent Ryff, On Muirhead's theorem...............

Carroll O. Wilde and Klaus G. Witz, Invariant means and the Stone-Čech compactification 